\title{
Estudo de Validação do Inventário de Personalidade Narcísica - 13 numa Amostra Escolar de Jovens Portugueses
}

\author{
Validation study of the Narcissistic Personality Inventory - 13 among a school \\ sample of Portuguese youths
}

\author{
Pedro Pechorro ${ }^{1}$, Cristina Nunes ${ }^{2}$, Rui Abrunhosa Gonçalves ${ }^{3}$, Mário R. Simões ${ }^{4}$ e João Pedro \\ Oliveira $^{5}$
}

\begin{abstract}
Resumo
O objetivo do presente estudo consistiu em traduzir, adaptar e validar o Inventário de Personalidade Narcísica-13 (NPI-13) numa amostra escolar $(N=543)$ de jovens portugueses de ambos os sexos. O NPI-13 demonstrou adequadas propriedades psicométricas, nomeadamente em termos da sua estrutura fatorial, consistência interna, validade convergente, validade discriminante e validade critério. Foram encontradas correlações positivas com sintomas de Perturbação do Comportamento, consumo de álcool, consumo de drogas e sexo sem proteção. As propriedades psicométricas encontradas legitimam na generalidade a utilização do NPI-13 com jovens portugueses em contexto escolar.
\end{abstract}

Palavras-chave: avaliação psicológica, NPI-13, narcisismo, adolescência

\begin{abstract}
The main aim of the present study was to translate and validate the Narcissistic Personality Inventory-13 (NPI-13) among a school sample $(N=543)$ of male and female Portuguese youths. The NPI-13 demonstrated good psychometric properties, namely in terms of its three-factor structure, internal consistency, convergent validity, discriminant validity and criterion-related validity. Positive correlations were found with Conduct Disorder symptoms, alcohol use, drug use and unprotected sex. The psychometric properties of the NPI-13 mostly justify its use among Portuguese adolescents in a school context.
\end{abstract}

Keywords: psychological assessment, NPI-13, narcissism, adolescence

A presente investigação foi financiada pela FCT (Bolsa SFRH/BPD/86666/2012), tendo decorrido no Centro de Investigação em Psicologia (CIPsi) da Escola de Psicologia da Universidade do Minho

${ }^{1}$ Escola de Psicologia, Universidade do Minho, Campus de Gualtar, 4710-057 Braga, Portugal. Tel.: 253604267. E-mail: ppechorro@gmail.com

${ }_{2}^{2}$ Centro de Investigação em Psicologia (CIP), Universidade do Algarve. Campus de Gambelas. 8005-139 Faro. Portugal. Tel.: 289800900. E-mail: csnunes@ualg.pt

${ }^{3}$ Escola de Psicologia, Universidade do Minho, Campus de Gualtar, 4710-057 Braga, Portugal. Tel.: 253604267. E-mail: rabrunhosa@psi.uminho.pt

${ }^{4}$ PsyAssessmentLab, Faculdade de Psicologia e de Ciências da Educação, Universidade de Coimbra. Rua do Colégio Novo, 3000 115 Coimbra, Portugal. Tel.: 239851450. E-mail: simoesmr@ fpce.uc.pt

${ }^{5}$ Universidade Lusófona de Humanidades e Tecnologias, Campo Grande 376, 1749-024 Lisboa, Portugal. Tel.: 217515500. E-mail: joaopoliveira@yahoo.com 


\section{Introdução}

O narcisismo é um constructo de enorme importância para a prática clínica, para a prática forense, para a prática educacional e para a investigação. De acordo com o Manual de Diagnóstico e Estatística das Perturbações Mentais - 5 (DSM-5; American Psychiatric Association, 2013), as características centrais do narcisismo incluem um sentimento grandioso de autoimportância, a necessidade de admiração excessiva, o sentimento de titularidade, a arrogância, a sensação de singularidade, a ausência de empatia, a inveja e a tendência para explorar as outras pessoas.

O constructo de narcisismo é tipicamente entendido como um traço de personalidade que varia num contínuo (Foster \& Campbell, 2007) e é caraterizado por um sentimento grandioso de si e um estilo interpessoal dominante e antagonista. As evidências científicas sugerem que se trata de um constructo heterogéneo composto por dimensões de grandiosidade e vulnerabilidade (Dickinson \& Pincus, 2003; Fossati et al., 2005; Miller \& Campbell, 2008; Miller, Hoffman, et al., 2011; Russ, Shedler, Bradley, \& Westen, 2008; Wink, 1991). Este traço de personalidade é mais prevalente entre os homens (Foster, Campbell, \& Twenge, 2003; Robins \& Trzesniewski, 2005; Stinson et al., 2008) e em grupos etários mais jovens (Foster et al., 2003; Wilson \& Sibley, 2011). Embora o narcisismo possa trazer alguns benefícios sociais a curto prazo (Campbell, Bush, Brunell, \& Shelton, 2005; Campbell \& Foster, 2007), a longo prazo, tem mais consequências nocivas e está associado a comportamentos interpessoais negativos (e.g., Bushman \& Baumeister, 1998; Campbell, Foster, \& Finkel, 2002; Nevicka, Ten Velden, De Hough, \& Van Vianen, 2011; Ryan, Weikel, \& Sprechini, 2008).

As evidências empíricas sugerem que $o$ narcisismo pode ser uma característica central subjacente ao comportamento antissocial entre os jovens. A pesquisa encontrou consistentemente uma associação entre narcisismo e comportamentos antissociais (e.g., Barry, Grafeman, Adler, \& Pickard, 2007, Chabrol, Van Leeuwen, Rodgers, \& Sejourne, 2009), problemas comportamentais, agressão proativa e baixa autoestima (e.g., Lau \& Marsee, 2013; Muñoz,
Kimonis, Frick, \& Aucoin, 2013; Washburn, McMahon, King, Reinecke, \& Silver, 2004). Sendo assim, não é surpreendente que se procure perceber melhor as implicações do narcisismo nos comportamentos agressivos, problemáticos e mal adaptativos entre os jovens (Auné, Facundo, \& Attorresi, 2017; Barry, Kerig, Stellwagen, \& Barry, 2011; Barry \& Kauten, 2014; Kauten, Barry, \& Leachman, 2013).

$\mathrm{O}$ instrumento de autorrelato mais usado para avaliar níveis subclínicos de narcisismo é o Inventário de Personalidade Narcísica (NPI; Raskin \& Hall, 1979), que é composto por 80 itens com base nos critérios do DSM-III para a perturbação narcísica da personalidade. Uma versão mais curta de 40 itens foi introduzida posteriormente (Raskin \& Terry, 1988). Este inventário avalia o narcisismo num contínuo, em que manifestações extremas representam o narcisismo patológico e formas menos extremas refletem o narcisismo como característica de personalidade (Emmons, 1987). O NPI tem sido investigado extensivamente desde o ponto de vista psicométrico (Emmons, 1984; Raskin \& Terry, 1988; Rhodewalt \& Morf, 1995). Neste plano existem questões em aberto relativamente à sua estrutura fatorial (e.g., Brown, Budzek, \& Tamorksi, 2009). Além disso, a sua eficiência em situações de pesquisa em que a pressão do tempo e a fadiga dos respondentes constituem fatores relevantes (Ames, Rose, \& Anderson, 2006).

Uma forma reduzida do NPI particularmente promissora foi recentemente desenvolvida, o NPI13 (Gentile et al., 2013), procurando colmatar as limitações mencionadas anteriormente. Gentile e colegas (2013) desenvolveram o NPI-13 especificamente para preservar os três fatores da estrutura obtida por Ackerman et al. (2011): Liderança/Autoridade

(L/A), Grandiosidade/Exibicionismo (G/E), e Empossamento/Exploratividade (E/E). A estrutura de três fatores do NPI-13 foi apoiada por análises fatoriais confirmatórias tendo demonstrado um melhor ajustamento do que os modelos de um e dois fatores (Gentile et al., 2013). Revelou ainda adequadas características psicométricas, a saber, consistência interna e validade de critério (Gentile et al., 2013). Em Portugal o NPI-13 foi validado utilizando amostras forenses juvenis masculinas e femininas (Pechorro, Gentile, Ray, Nunes, \& 
Gonçalves, 2016; Pechorro, Maroco, Ray, Gonçalves, \& Nunes, no prelo), tendo a análise fatorial confirmatória identificado a esperada estrutura de três fatores. $\mathrm{O}$ instrumento demonstrou também propriedades psicométricas aceitáveis em termos de consistência interna, validade convergente, validade discriminante e validade de critério.

Dado que o narcisismo é um traço de personalidade particularmente relevante para a compreensão do ajustamento individual e social dos jovens, consideramos ser importante a disponibilização dum instrumento breve, psicometricamente adequado e devidamente validado para os adolescentes Portugueses em contexto escolar. Desta forma, o objetivo da presente investigação foi a validação da versão portuguesa do NPI-13 em uma amostra escolar de jovens portugueses de ambos os sexos. Colocámos as hipóteses de que a versão portuguesa do NPI-13 demonstrará: (1) uma estrutura tridimensional; (2) adequada consistência interna; (3) validade convergente com as medidas de avaliação da psicopatia, da agressividade e de impulsividade, e validade discriminante com a medida de ansiedade social; (4) validade de critério (ou concorrente) com sintomas de perturbação de comportamento, consumo de álcool, de drogas e sexo sem preservativo.

\section{Método}

\section{Participantes}

A amostra composta por 543 participantes $(M=15.65$ anos; $D P=1.84$ anos; amplitude $=12-20$ anos), provenientes de estabelecimentos públicos de ensino da grande Lisboa, Algarve e Coimbra, foi subdividida em rapazes $(n=269 ; M=15.80$ anos; $D P=1.81$ anos; amplitude $=12-20$ anos) e raparigas $(n=274 ; M=15.50$ anos; $D P=1.86$ anos; amplitude $=12-20$ anos). Não foram encontradas diferenças estatisticamente significativas entre rapazes e raparigas relativamente à idade $(F=3.52$; $p=.06)$, anos de escolaridade completados $(F=5.53 ; p=.12)$ e etnia $\left(\chi^{2}=6.69, p=.12\right)$.

\section{Medidas}

O Inventário de Personalidade Narcísica - 13 (NPI-13; Gentile et al., 2013) é uma versão breve composta por itens dicotómicos do NPI (Raskin \& Terry, 1988), considerado a medida mais utilizada para medir o constructo de narcisismo. Gentile et al. (2013) desenvolveram o NPI-13 com o intuito de preservar a estrutura de três fatores conceptualizada por Ackerman et al. (2011), nomeadamente Liderança/Autoridade (L/A), Grandiosidade/Exibicionismo (G/E), e Empossamento/Exploratividade (E/E). $\quad \mathrm{Na}$ presente investigação foi utilizada a versão portuguesa do NPI-13 (Pechorro, Gentile, et al., 2016).

O Inventário de Traços Psicopáticos para Jovens (Youth Psychopathic Traits Inventory Short version - YPI-S; van Baardewijk et al., 2010) foi concebido para medir traços psicopáticos em adolescentes. Os 18 itens que o constituem são cotados numa escala ordinal de 4 pontos, de Não se aplica a Aplica-se muito bem. $\mathrm{O}$ YPI-S tem uma estrutura tridimensional semelhante à do YPI original composta pelas dimensões comportamental, afetiva e interpessoal. Pontuações mais elevadas indicam maior presença dos traços em questão. Na presente investigação foi utilizada a versão portuguesa do YPI-S (Pechorro, Ribeiro da Silva, Andershed, Rijo, \& Gonçalves, 2016; Pechorro, Gonçalves, Andershed, \& DeLisi, 2017). O coeficiente alfa do YPI-S na presente investigação foi .92.

O Questionário de Agressividade ReativaProativa (Reactive-Proactive Aggression Questionnaire - RPQ; Raine et al., 2006) é uma medida de autorresposta constituída por 23 itens que distingue entre agressividade reativa $\mathrm{e}$ proativa. Cada item é cotado em escala ordinal de 3 pontos, de Nunca a Frequentemente. O RPQ pode ser utilizado com adolescentes e jovens adultos. Pontuações mais elevadas indicam maior presença dos traços em questão. Na presente investigação foi utilizada a versão portuguesa do RPQ (Pechorro, Kahn, Ray, Raine, \& Gonçalves, 2017; Pechorro, Ayala-Nunes, Kahn, \& Nunes, no prelo). O coeficiente alfa do RPQ na presente investigação foi .86 .

A Escala de Impulsividade de Barratt - versão 11 (Barratt Impulsiveness Scale version 11 - BIS11; Patton et al., 1995) é uma escala de autorresposta composta por 30 itens projetada para medir impulsividade. Os itens são cotados numa escala ordinal de 4 pontos, de 
Raramente/Nunca a Quase sempre/Sempre. A BIS-11 tem seis subescalas que correspondem a seis fatores de primeira ordem, nomeadamente: Atenção (5 itens), Instabilidade cognitiva (3 itens), Motora (7 itens), Perseverança (4 itens), Autocontrolo (6 itens) e Complexidade cognitiva (5 itens) que deverão convergir em três fatores de segunda ordem, nomeadamente: Impulsividade atencional, Impulsividade motora e Impulsividade de não-planeamento. Pontuações mais elevadas indicam maior presença de impulsividade. $\mathrm{Na}$ presente investigação utilizou-se a versão portuguesa da BIS-11 (Pechorro, Ayala-Nunes, Ray, Nunes, \& Gonçalves, 2016), mais especificamente a versão reduzida composta pelos 18 itens dos fatores de primeira ordem Atenção, Motora e Autocontrolo (Pechorro, Oliveira, Gonçalves, \& Jesus, no prelo). O coeficiente alfa da BIS-11 versão reduzida na presente investigação foi .87 .

A Escala de Ansiedade Social para Adolescentes (Social Anxiety Scale for Adolescents - SAS-A; La Greca \& Lopez, 1998) é uma medida de autorresposta constituída por 22 itens (dos quais 4 são itens neutros não cotados) que avalia as experiências de ansiedade social dos adolescentes no contexto das relações com os seus pares. Os itens são avaliados segundo uma escala ordinal de 5 pontos, que vai De forma nenhuma a Todas as vezes. Para além da pontuação total pode-se obter também as pontuações nas três subescalas, nomeadamente: FNE, SAD-Novo e SAD-Geral (La Greca \& Lopez, 1998). Pontuações mais elevadas indicam níveis de ansiedade social mais elevados. $\mathrm{Na}$ presente investigação foi utilizada a versão portuguesa da SAS-A (Pechorro, Ayala-Nunes, Nunes, Maroco, \& Gonçalves, 2016). O coeficiente alfa da SAS-A na presente investigação foi .92 .

Os 15 critérios para o diagnóstico de Perturbação do Comportamento de acordo com os critérios oficiais do DSM-5 (American Psychiatric Association, 2013) foram utilizados para criar uma escala de autorresposta (Skilling, Quinsey, \& Craig, 2001). Os 15 itens (codificados 0=Não, $1=$ Sim) foram somados para obter uma pontuação total, de forma que pontuações mais altas indicam maior presença de sintomas de perturbação de comportamento. $\mathrm{O}$ coeficiente alfa na presente investigação foi .81 .
Foi construído um questionário ad hoc para descrever as caraterísticas sociodemográficas dos participantes, que incluiu variáveis como idade, sexo, escolaridade e etnia. Este questionário incluiu também itens ordinais tipo Likert de 5 pontos sobre consumo de álcool, drogas leves (cannabis), drogas duras (cocaína, heroína) e sexo desprotegido (i.e., sem preservativo).

\section{Procedimentos}

Pediu-se previamente autorização à autora do NPI-13 (Gentile et al., 2013) para traduzir e utilizar o instrumento em Portugal. Durante o processo de tradução e adaptação do NPI-13 seguiram-se recomendações estabelecidas internacionalmente (Hambleton, Merenda, \& Spielberger, 2005). O primeiro autor deste artigo e um colega efetuaram a tradução da escala. De seguida um tradutor bilingue fez a respetiva retroversão para inglês, que foi então comparada com o instrumento original. Foi então realizado um estudo pré-teste para analisar a qualidade da tradução, detetar eventuais problemas e aperfeiçoar a linguagem de forma a torná-la mais facilmente entendível. Chegou-se assim à versão final da escala (para uma descrição mais detalhada ver Pechorro, Gentile, et al., 2015).

O procedimento de recolha dos questionários decorreu em escolas básicas/secundárias da região de Lisboa, Algarve e Coimbra após se ter obtido autorização por parte da Direção-Geral de Educação (DGE). Foi entregue um termo de consentimento assinado pelo encarregado de educação de cada aluno autorizando a participação na investigação. A aplicação decorreu em contexto de grupo. A taxa de participação foi de aproximadamente $85 \%$. Foram excluídos os participantes que estavam fora do intervalo etário estabelecido ou que entregaram questionários não preenchidos, incompletos ou ilegíveis.

Os dados foram inseridos $\mathrm{e}$ analisados utilizando o software SPSS v25 (IBM SPSS, 2017) e o software EQS 6.3 (Bentler \& Wu, 2015). No tratamento de dados estatísticos recorreu-se a estatísticas descritivas, ANOVA, qui-quadrado, análise fatorial confirmatória, análise de consistência interna por alfa de Cronbach e correlações paramétricas e nãoparamétricas. 
A análise da estrutura fatorial da versão reduzida do NPI-13 foi efetuada no software EQS. Os índices de ajustamento calculados incluíram: Qui-quadrado de Satorra-Bentler, CFI (Comparative Fit Index), IFI (Incremental Fit Index), RMSEA (Root Mean Square Error of Approximation). Valores CFI $\geq .90$ e RMSEA $\leq .10$ indicam ajustamento adequado; valores de CFI $\geq .95$ e RMSEA $\leq .06$ indicam um ajustamento bom (Byrne, 2006). Um valor de IFI $\geq .90$ é considerado aceitável. Foram utilizados Índices de Modificação para melhorar o ajustamento do modelo. A AFC foi efetuada diretamente nos itens utilizando valores de cargas fatoriais $\geq .30$. Optouse pela utilização de uma matriz de correlações policóricas com métodos de estimação robustos nos itens ordinais dado que proporcionam melhores resultados (Byrne, 2006).

Foram utilizadas correlações Pearson para analisar as associações entre variáveis escalares e correlações Spearman para analisar a relação entre variáveis ordinais e variáveis escalares (Leech, Barrett, \& Morgan, 2015). Em termos de magnitude de correlações, consideraram-se correlações fracas as correlações entre 0 e .20, correlações moderadas entre .20 e .50 , e correlações fortes acima de .50 (Ferguson, 2009). A consistência interna por alfa de Cronbach foi considerada marginalmente aceitável se entre .60 e .69, aceitável se entre .70 e .79 e boa se acima de .80 (DeVellis, 1991; Nunnally \& Bernstein, 1994).

\section{Resultados}

O primeiro passo do tratamento de dados consistiu na verificação da estrutura fatorial de três fatores. Os seguintes índices de ajustamento foram obtidos para a amostra total: $\mathrm{S}-\mathrm{B} \chi^{2}=393.83$, $p \leq .001 ; \mathrm{IFI}=.94$; CFI=.94; RMSEA=.09 (.08-.10). Foram utilizados índices de modificação (entre o item 3 item 5, entre o item 1 e o item 2). No Quadro 1 podem-se observar as saturações fatoriais dos itens e as correlações item-total corrigidas.

No Quadro 2 são apresentadas as correlações entre as dimensões do NPI-13 e os valores de consistência interna.

No Quadro 3 são apresentadas a validade convergente, discriminante e de critério. A validade convergente foi efetuada com o YPI-S, o RPQ e a BIS-11 versão reduzida. A validade discriminante foi efetuada com a SAS-A. A validade de critério (ou concorrente) foi efetuada com os sintomas de Perturbação de Comportamento do DSM-5 (American Psychiatric Association, 2013), o consumo de álcool, de cannabis, de drogas duras e os comportamentos sexuais desprotegidos (i.e., sexo sem preservativo).

Quadro 1. Saturações fatoriais e correlações item-total para o modelo tridimensional do NPI-13

\begin{tabular}{lcc}
\hline Itens & Saturações & CITC \\
\hline Liderança/Autoridade (L/A) & & \\
1. Gosto de ter autoridade sobre outras pessoas. & .83 & .60 \\
2. Tenho uma vontade forte de ser eu a mandar. & .64 & .59 \\
3. As pessoas parecem sempre reconhecer a minha autoridade. & .57 & .54 \\
4. Ser líder está na minha natureza. & .64 & .61 \\
Grandiosidade/Exibicionismo (G/E) & & \\
5. Eu sei que sou bom porque as pessoas me dizem isso. & .46 & .44 \\
6. Gosto de exibir o meu corpo. & .85 & .51 \\
7. Gosto de ver o meu corpo. & .94 & .61 \\
8. Gosto de me exibir quando tenho oportunidade. & .74 & .58 \\
9. Gosto de me olhar no espelho. & .34 & .27 \\
Empossamento/Exploratividade (E/E) & & \\
10. É fácil para mim manipular as outras pessoas. & .67 & .45 \\
11. Insisto em que as pessoas me respeitem. & .41 & .36 \\
12. Sou muito exigente com as outras pessoas. & .62 & .47 \\
13. Só ficarei satisfeito quando tiver tudo a quanto tenho direito. & .78 & .57
\end{tabular}

Nota. NPI-13=Inventário de Personalidade Narcísica-13, M/F=Masculino/Feminino, CITC=Correlações item-total corrigidas 
Quadro 2. Matriz de correlações e consistência interna do NPI-13

\begin{tabular}{|c|c|c|c|c|}
\hline & NPI-13 total & $\mathrm{L} / \mathrm{A}$ & $\mathrm{G} / \mathrm{E}$ & $\mathrm{E} / \mathrm{E}$ \\
\hline \multicolumn{5}{|l|}{ Masculino } \\
\hline NPI-13 total & 1 & & & \\
\hline $\mathrm{L} / \mathrm{A}$ & $.84 * * *$ & 1 & & \\
\hline $\mathrm{G} / \mathrm{E}$ & $.80 * * *$ & $.50 * * *$ & 1 & \\
\hline $\mathrm{E} / \mathrm{E}$ & $.85 * * *$ & $.68 * * *$ & $.44 * * *$ & 1 \\
\hline$\alpha$ Cronbach & .83 & .64 & .72 & .63 \\
\hline MCII & .30 & .37 & .39 & .31 \\
\hline \multicolumn{5}{|l|}{ Feminino } \\
\hline NPI-13 total & 1 & & & \\
\hline $\mathrm{L} / \mathrm{A}$ & $.87 * * *$ & 1 & & \\
\hline $\mathrm{G} / \mathrm{E}$ & $.78 * * *$ & $.53 * * *$ & 1 & \\
\hline $\mathrm{E} / \mathrm{E}$ & $.64 * * *$ & $.64 * * *$ & $.42 * * *$ & 1 \\
\hline$\alpha$ Cronbach & .82 & .74 & .68 & .61 \\
\hline MCII & .29 & .48 & .34 & .27 \\
\hline
\end{tabular}

Nota. NPI-13=Inventário de Personalidade Narcísica-13, L/A=Liderança/Autoridade, G/E=Grandiosidade/Exibicionismo, $\mathrm{E} / \mathrm{E}=$ Empossamento/Exploratividade, $\alpha$ Cronbach=Alfa de Cronbach, MCII=Médias das correlações inter-itens $* * * p<.001$

Quadro 3. Validade convergente, discriminante e de critério do NPI-13

\begin{tabular}{|c|c|c|c|c|}
\hline & Total & $\mathrm{L} / \mathrm{A}$ & G/E & $\mathrm{E} / \mathrm{E}$ \\
\hline \multicolumn{5}{|l|}{ Masculino } \\
\hline YPI-S & $.44 * * *$ & $.41 * * *$ & $.24 * * *$ & $.42 * * *$ \\
\hline RPQ & $.41 * * *$ & $.38 * * *$ & $.25 * * *$ & $.36 * * *$ \\
\hline BIS-11 & $.15^{* *}$ & $.13^{*}$ & $.14^{*}$ & .08 \\
\hline SAS-A & -.10 & -.10 & -.06 & -.08 \\
\hline Sintomas PC & $.23 * * *$ & $.24 * * *$ & $.11 *$ & $.24 * * *$ \\
\hline Álcool & $.24 * * *$ & $.22 * * *$ & $.15 * *$ & $22 * * *$ \\
\hline Cannabis & $.12 *$ & .08 & .07 & $.15^{* *}$ \\
\hline Drogas duras & $.19 * * *$ & $.17 * *$ & .04 & $.27 * * *$ \\
\hline Sexo desprot. & $.17 * *$ & $.16^{* *}$ & .08 & $.17 * *$ \\
\hline \multicolumn{5}{|l|}{ Feminino } \\
\hline YPI-S & $.48 * * *$ & $.44 * * *$ & $.27 * * *$ & $.42 * * *$ \\
\hline RPQ & $.33 * * *$ & $.30 * * *$ & $.15^{* *}$ & $.32 * * *$ \\
\hline BIS-11 & $.19 * * *$ & $.16^{* *}$ & $.11 *$ & $.19 * * *$ \\
\hline SAS-A & $-.12 *$ & $-.11 *$ & $-.11 *$ & -.04 \\
\hline Sintomas PC & $.12 *$ & $.16^{* *}$ & .01 & .09 \\
\hline Álcool & $.24 * * *$ & $.19 * * *$ & $.17 * *$ & $.22 * * *$ \\
\hline Cannabis & .08 & .03 & .02 & $.18 * * *$ \\
\hline Drogas duras & .09 & $.11 *$ & .02 & .07 \\
\hline Sexo desprot. & $.14^{*}$ & .05 & .08 & $.20 * * *$ \\
\hline
\end{tabular}

Nota. NPI-13=Inventário de Personalidade Narcísica-13, YPI-S=Inventário de Traços Psicopáticos para Jovens - versão reduzida, RPQ=Questionário de Agressividade Reativa-Proactiva, SAS-A=Escala de Ansiedade Social para Adolescentes, Sintomas $\mathrm{PC}=$ Sintomas de Perturbação do Comportamento, Sexo desprot.=Sexo sem preservativo $* * * p<.001 ; * * p<.01 ; * p<.05$

\section{Discussão}

O objetivo da presente investigação foi a adaptação de uma versão curta do NPI-13 em adolescentes portugueses. Os resultados obtidos neste estudo revelaram a existência de uma estrutura fatorial de três fatores com aceitáveis índices de ajustamento (Maroco, 2014; West et al., 2012). De acordo com o esperado, as correlações entre o NPI-13 e as suas dimensões quer para o grupo masculino, quer para o grupo feminino, apresentaram associações positivas que variaram de moderadas a altas. A nossa primeira hipótese foi portanto confirmada.

A análise da consistência interna do NPI-13 através de alfa de Cronbach, revelou valores bons, sempre superiores a .70 para a escala total (Nunnally \& Bernstein, 1994). Todavia, na 
amostra masculina as subescalas L/A e E/E e na amostra feminina as subescalas $\mathrm{G} / \mathrm{E}$ e $\mathrm{E} / \mathrm{E}$ obtiveram valores marginais, entre .60 e .70 . Estes resultados recomendam alguma precaução na utilização destas subescalas. As médias das correlações inter-itens e a amplitude de correlações item-total corrigidas (Clark \& Watson, 1995) revelaram predominantemente valores adequados (amplitude de .15 a $.50 \mathrm{e}$ correlações acima de .20, respetivamente). Portanto, a nossa segunda hipótese foi também confirmada.

A validade convergente (Urbina, 2014) do NPI-13 com o YPI-S, o RPQ e a BIS-11 revelou de uma forma global correlações positivas baixas a moderadas estatisticamente significativas conforme o esperado, em linha com investigações prévias (e.g., Barry, Frick, \& Killian, 2003; Feilhauer, Cima, \& Arntz, 2012; Pechorro et al., 2016). A validade discriminante com a SAS-A revelou as esperadas correlações negativas ou não-significativas (Kaplan \& Saccuzzo, 2013). Desta forma consideramos que a terceira hipótese foi confirmada.

A validade de critério (ou concorrente) do NPI-13 com sintomas de perturbação de comportamento foram de uma forma geral encontradas as correlações positivas estatisticamente significativas esperadas, embora estas tenha sido mais fracas no caso do sexo feminino. No que diz respeito às variáveis consumo de álcool, consumo de cannabis e sexo sem preservativo foram encontradas principalmente correlações baixas ainda que estatisticamente significativas (e.g., Carvalho \& Novo, 2014; Stinson et al., 2008). Todavia, a exceção foi o uso de cannabis e de drogas duras entre o sexo feminino que revelou correlações não-significativas. A última hipótese foi também confirmada.

A título de conclusão, pensamos que o NPI-13 demonstrou propriedades psicométricas na generalidade aceitáveis que justificam a sua utilização com adolescentes portugueses em contexto escolar. Os benefícios derivados da utilização desta medida em contexto educativo são consideráveis. Por exemplo: poderá servir para identificar alunos com elevado narcisismo que poderão beneficiar de intervenções orientadas no sentido de prevenir eventuais padrões comportamentais problemáticos, agressivos e mal adaptativos (Washburn et al., 2004). Sendo o narcisismo um traço da personalidade de enorme importância a nível clínico, forense, educacional e de investigação, a adaptação nacional do NPI-13 vem contribuir para colmatar a necessidade de medidas de avaliação de narcisismo aplicáveis especificamente a adolescentes (Pechorro et al., 2016).

Importa reconhecer algumas limitações da presente investigação. Neste plano, teria sido útil a utilização de outras medidas validadas de narcisismo em adolescentes portugueses para analisar a validade convergente (e.g., com o NPI original). É igualmente necessária precaução relativamente à generalização dos resultados para outras amostras (e.g., validação cruzada em amostras clínicas). Estudos futuros devem ser realizados com vista à obtenção de mais dados de natureza psicométrica sobre o instrumento (e.g., estudos de validação cruzada, precisão testereteste, invariância de medida). Seria também interessante utilizar escalas tipo Likert em vez de itens dicotómicos, o que poderia melhorar os valores de consistência interna. Esperamos que este trabalho possa contribuir para o enriquecimento das conceptualizações científicas que se encontram em curso sobre narcisismo, no contexto da realização de novos trabalhos de investigação.

\section{Referências}

American Psychiatric Association. (2013). Diagnostic and statistical manual of mental disorders ( $5^{\text {th }}$ ed.). Arlington, VA: American Psychiatric Publishing.

Auné, S., Facundo, A., \& Attorresi, H. (2017). Propiedades psicométricas de una prueba de conducta empática. Revista Iberoamericana de Diagnóstico y Evaluación - e Avaliação Psicológica, 45, 47-56. doi: 10.21865/RIDEP45.3.04

Barry, C. T., Frick, P. J., \& Killian, A. L. (2003). The relation of narcissism and self-esteem to conduct problems in children: A preliminary investigation. Journal of Clinical Child and Adolescent Psychology, 32(1), 139-152. doi: 10.1207/S15374424JCCP3201_13 
Barry, C. T., Grafeman, S. J., Adler, K. K., \& Pickard, J. D. (2007). The relations among narcissism, self-esteem, and delinquency in a sample of at-risk adolescents. Journal of Adolescence, $\quad 30, \quad 933-942$. doi:10.1016/j.adolescence.2006.12.003

Barry, C. T., \& Kauten, R. L. (2014). Nonpathological and pathological narcissism: Which self-reported characteristics are most problematic in adolescents? Journal of Personality Assessment, 96, 212-219. doi:10.1080/00223891.2013.830264

Barry, C. T., Kerig, P., Stellwagen, K., \& Tammy Barry, T. (Eds) (2011). Narcissism and machiavellianism in youth: Implications for the development of adaptive and maladaptive behavior. Washington, DC: American Psychological Association.

Bentler, P., \& Wu, E. (2015). Supplement to EQS 6.3 for Windows user's guide. Temple City, CA: Multivariate Software.

Brown, R. P., Budzek, K., \& Tamborski, M. (2009). On the meaning and measure of narcissism. Personality and Social Psychology Bulletin, 35, 951-964. doi: $10.1177 / 0146167209335461$

Bushman, B. J., \& Baumeister, R. F. (1998). Threatened egotism, narcissism, self-esteem, and direct and displaced aggression: Does self-love or self-hate lead to violence? Journal of Personality and Social Psychology, 75(1), 219-229. doi:10.1037/0022-3514.75.1.219

Byrne, B. (2006). Structural equation modeling with EQS: Basic concepts, applications, and programming. Mahwah, NJ: Lawrence Erlbaum Associates.

Campbell, W. K., Bush, C. P., Brunell, A. B., \& Shelton J. (2005). Understanding the social costs of narcissism: The case of the tragedy of the commons. Personality \& Social Psychology Bulletin, 10, 1358-1368. doi: $10.1177 / 0146167205274855$

Campbell, W. K., \& Foster, J. D. (2007). The narcissistic self: Background, an extended agency model, and ongoing controversies. In C. Sedikides \& S. Spencer (Eds.), Frontiers in social psychology: The self (pp. 115-138). Philadelphia, PA: Psychology Press.

Campbell, W. K., Foster, C. A., \& Finkel, E. J. (2002). Does self-love lead to love for others?
A story of narcissistic game playing. Journal of Personality and Social Psychology, 83, 340-354. doi:10.1037/0022-3514.83.2.340

Carvalho, R. G., \& Novo, R. F. (2014). Dimensões da personalidade e comportamentos de risco na adolescência: Um estudo com a versão portuguesa do MMPI-A. Revista Iberoamericana de Diagnóstico y Evaluación - e Avaliação Psicológica, 37(1), 203-222.

Chabrol, H., Van Leeuwen, N., Rodgers, R., \& Séjourné, N. (2009). Contributions of psychopathic, narcissistic, Machiavellian, and sadistic personality traits to juvenile delinquency. Personality and Individual Differences, $\quad 47, \quad 734-739$. doi:10.1016/j.paid.2009.06.020

Clark, L., \& Watson, D. (1995). Constructing validity: Basic issues in objective scale development. Psychological Assessment, 7, 309-319. doi:10.1037//1040-3590.7.3.309

DeVellis, R.F. (1991). Scale development: Theory and applications. Newbury Park: Sage Publications, Inc.

Dickinson, K. A., \& Pincus, A. L. (2003). Interpersonal analysis of grandiose and vulnerable narcissism. Journal of Personality Disorders, 17, 188-207. doi:10.1521/pedi.17.3.188.22146

Emmons, R. A. (1984). Factor analysis and construct validity of the Narcissistic Personality Inventory. Journal of Personality Assessment, $\quad 48, \quad 291-300$. doi:10.1207/s15327752jpa4803_11

Emmons, R. A. (1987). Narcissism: Theory and measurement. Journal of Personality and Social Psychology, 52, 11-17. doi:10.1037/0022-3514 .52.1.11

Feilhauer, J., Cima, M., \& Arntz, A. (2012). Assessing callous-unemotional traits across different groups of youths: Further crosscultural validation of the Inventory of Callous-Unemotional Traits. International Journal of Law and Psychiatry, 35, 251-262. doi:10.1016/j.ijlp.2012.04.002

Ferguson, C. (2009). An effect size primer: A guide for clinicians and researchers. Professional Psychology: Research and Practice, 40, 532-538. doi:10.1037/a0015808 
Fossati, A., Beauchaine, T. P., Grazioli, F., Carretta, I., Cortinovis, F., \& Maffei, C. (2005). A latent structure analysis of Diagnostic and Statistical Manual of Mental Disorders, fourth edition, narcissistic personality disorder criteria. Comprehensive Psychiatry, 46, 361-367. doi:10.1016/j.comppsych.2004.11.006

Foster, J. D., \& Campbell, W. K. (2007). Are there such things as "Narcissists" in social psychology? A taxometric analysis of the Narcissistic Personality Inventory. Personality and Individual Differences, 43, 1321-1332. doi:10.1016/j.paid.2007.04.003

Foster, J. D., Campbell, W. K., \& Twenge, J. M. (2003). Individual differences in narcissism: Inflated self-views across the lifespan and around the world. Journal of Research in Personality, 37, 469-486. doi:10.1016/S0092-6566(03)00026-6

Gentile, B., Miller, J., Hoffman, B., Reidy, D., Zeichner, A., \& Campbell, W. (2013). A test of two brief measures of grandiose narcissism: The Narcissistic Personality Inventory-13 and the Narcissistic Personality Inventory-16. Psychological Assessment, 25, 1120-1136. doi:10.1037/a0033192

Hambleton, R., Merenda, P., \& Spielberger, C. (2005). Adapting educational and psychological tests for cross-cultural assessment. Mahwah, NJ: Lawrence Erlbaum Associates.

IBM SPSS. (2017). IBM SPSS statistics base 25. Chicago, IL: SPSS.

Kaplan, R., \& Saccuzzo, D. (2013). Psychological testing: Principles, applications, and issues ( $8^{\text {th }}$ ed.). Belmont, CA: Wadsworth, Cengage Learning.

Kauten, R., Barry, C. T., \& Leachman, L. (2013). Do perceived social stress and resilience influence the effects of psychopathy-linked narcissism and $\mathrm{CU}$ traits on adolescent aggression? Aggressive Behavior, 39, 381390. doi:10.1002/Ab.21483

La Greca, A., \& Lopez, N. (1998) Social anxiety among adolescents: Linkages with peer relations and friendships. Journal of Abnormal Child Psychology, 26, 83-94. doi:10.1023/A:1022684520514
Lau, K. S., \& Marsee, M. A. (2013). Exploring narcissism, psychopathy, and Machiavellianism in youth: Examination of associations with antisocial behavior and aggression. Journal of Child and Family Studies, 22, 355-367. doi:10.1007/s10826-012-9586-0

Leech, N., Barrett, K., \& Morgan, G. (2015). IBM SPSS for intermediate statistics. New York, NY:LEA.

Lynam, D. R. (2011). Psychopathy and Narcissism. In W. K. Campbell \& J. D. Miller (Eds.), The handbook of narcissism and narcissistic personality disorder: Theoretical approaches, empirical findings, and treatments (pp. 272-282). New Jersey: John Wiley \& Sons.

Maroco, J. (2014). Análise de equações estruturais: Fundamentos teóricos, software \& aplicações [Structural equations analysis: Theoretical foundations, software and applications]. Pero Pinheiro: ReportNumber.

Miller, J. D., \& Campbell, W. K. (2008). Comparing clinical and social personality conceptualizations of narcissism. Journal of Personality, 76, 449-476. doi:10.1111/j.1467-6494.2008.00492.x

Miller, J. D., Hoffman, B. J., Gaughan, E. T., Gentile, B., Maples, J., \& Campbell, W. K. (2011). Grandiose and vulnerable narcissism: A nomological network analysis. Journal of Personality, $\quad 79, \quad 1013-1042$. doi:10.1111/j.1467-6494.2010.00711.x

Muñoz, L. C., Kimonis, E. R., Frick, P. J., \& Aucoin, K. J. (2013). Emotional reactivity and the association between psychopathy-linked narcissism and aggression in detained adolescent boys. Development and Psychopathology, 25, 473-485. doi:10.1017/S0954579412001186

Nevicka, B., Ten Velden, F. S., De Hoogh, A. H. B., \& Van Vianen, A. E. M. (2011). Reality at odds with perceptions: Narcissistic leaders and group performance. Psychological Science, 22, 1259-1264. doi:10.1177/0956797611417259

Nunnally, J. C., \& Bernstein, I. H. (1994). Psychometric theory ( $3^{\text {rd }}$ ed.). New York: McGraw-Hill.

Patton, J., Stanford, M., \& Barratt, E. (1995). Factor structure of the Barratt Impulsiveness 
Scale. Journal of Clinical Psychology, 51, 768-774. doi:10.1002/1097-4679(199511)51:6<768::AIDJCLP2270510607>3.0.CO;2-1

Pechorro, P., Ribeiro da Silva, D., Rijo, D., Gonçalves, R. A., \& Andershed, H. (2017). Psychometric properties and measurement invariance of the Youth Psychopathic Traits Inventory - Short among Portuguese youths. Journal of Psychopathology and Behavioral Assessment, $\quad 39, \quad 486-497$. doi:10.1007/s10862-017-9597-7

Pechorro, P., Gonçalves, R., Andershed, H., \& DeLisi, M. (2017). Female psychopathic traits in forensic and school context: Comparing the Antisocial Process Screening Device SelfReport and Youth Psychopathic Traits Inventory Short version among Portuguese youth. Journal of Psychopathology and Behavioral Assessment, 39, 642-665. doi:10.1007/s10862-017-9605-y

Pechorro, P., Kahn, R., Ray, J., Raine, A., \& Gonçalves, R. A. (2017). Psychometric properties of the Reactive-Proactive Aggression Questionnaire among detained female juvenile delinquents and community youths. Criminal Justice and Behavior, 44, 531-550. doi:10.1177/0093854816686395

Pechorro, P., Ayala-Nunes, L., Kahn, R., \& Nunes, C. (no prelo). The Reactive-Proactive Aggression Questionnaire: Measurement invariance and reliability among a school sample of Portuguese youths. Child Psychiatry \& Human Development. doi:10.1007/s10578-017-0772-6

Pechorro, P., Ayala-Nunes, L., Ray, J., Nunes, C., \& Gonçalves, R. (2016). Psychometric properties of the Portuguese version of the Barratt Impulsiveness Scale - 11 among a school sample of male and female adolescents. Journal of Child and Family Studies, 25, 2753-2764. doi:10.1007/s10826-016-0453-2

Pechorro, P., Oliveira, J.P., Gonçalves, R., \& Jesus, S. (no prelo). Propriedades psicométricas de uma versão reduzida da Escala de Impulsividade de Barratt - 11 numa amostra escolar de adolescentes portugueses. Revista Iberoamericana de Diagnóstico y Evaluación - e Avaliação Psicológica.
Pechorro, P., Gentile, B., Ray, J. V., Nunes, C., \& Gonçalves, R. (2016). Adaptation of the Narcissistic Personality Inventory among a Portuguese sample of incarcerated juvenile offenders. Psychology, Crime \& Law, 22, 495511. doi:10.1080/1068316X.2016.1168421

Pechorro, P., Maroco, J., Ray, J. V., Gonçalves, R., \& Nunes, C. (no prelo). A brief measure of narcissism among female juvenile delinquents and community youths: The Narcissistic Personality Inventory-13. International Journal of Offender Therapy and Comparative Criminology. doi:10.1177/0306624X17700855

Raine, A., Dodge, K., Loeber, R., Gatzke-Kopp, L., Lynam, D., Reynolds, C., ... Liu, J. (2006). The Reactive-Proactive Aggression Questionnaire: Differential correlates of reactive and proactive aggression in adolescent boys. Aggressive Behavior, 32, 159-171. doi:10.1002/ab.20115

Raskin, R. N., \& Hall, C. S. (1979). A narcissistic personality inventory. Psychological Reports, 45, 590. doi:10.2466/pr0.1979.45.2.590

Raskin, R., \& Shaw, R. (1988). Narcissism and the use of personal pronouns. Journal of Personality, 56, 393-404. doi:10.1111/j.1467-6494.1988.tb00892.x

Raskin, R., \& Terry, H. (1988). A principalcomponents analysis of the Narcissistic Personality Inventory and further evidence of its construct validity. Journal of Personality and Social Psychology, 54, 890-902. doi:10.1037/0022-3514.54.5.890

Robins, R.W., \& Trzesniewski, K.H. (2005). Selfesteem development across the lifespan. Current Directions in Psychological Science, 14, 158-162. doi:10.1111/j.0963-7214.2005.00353.x

Rhodewalt, F., \& Morf, C. C. (1995). Self and interpersonal correlates of the Narcissistic Personality Inventory: A review and new findings. Journal of Research in Personality, 29, 1-23. doi:10.1006/jrpe.1995.1001

Russ, E., Shedler, J., Bradley, R., \& Westen, D. (2008). Refining the construct of narcissistic personality disorder: Diagnostic criteria and subtypes. The American Journal of Psychiatry, 165, 1473-1481. doi:10.1176/appi.ajp.2008.07030376 
Ryan, K. M., Weikel, K., \& Sprechini, G. (2008). Gender differences in narcissism and courtship violence in dating couples. Sex Roles, 58, 802-813. doi:10.1007/s11199-008-9403-9

Skilling, T., Quinsey, V., \& Craig, W. (2001). Evidence of a taxon underlying serious antisocial behavior in boys. Criminal Justice and Behavior, 28, 450-470. doi:10.1177/009385480102800404

Stinson, F. F., Dawson, D. A., Goldstein, R. B., Chou, S. P., Smith, S. M., Ruan, W. J. (...) Grant, B. F. (2008). Prevalence, correlates, disability and comorbidity of DSM-IV Narcissistic Personality Disorder: Results from the Wave 2 National Epidemiologic Survey on Alcohol and Related Conditions. Journal of Clinical Psychiatry, 69, 10331045. doi:10.4088/JCP.v69n0701

Urbina, S. (2014). Essentials of psychological testing $\left(2^{\text {nd }}\right.$ ed.). Hoboken, NJ: John Wiley \& Sons.

Van Baardewijk, Y., Andershed, H., Stegge, H., Nilsson, K., Scholte, E., \&Vermeiren, R. (2010). Development and tests of short versions of the Youth Psychopathic Traits Inventory and the Youth Psychopathic Traits Inventory-Child Version. European Journal of Psychological Assessment, 26, 122-128. doi:10.1027/1015-5759/a000017

Washburn, J., McMahon, S., King, C., Reinecke, M., \& Silver, C. (2004). Narcissistic features in young adolescents: Relations to aggression and internalizing symptoms. Journal of Youth and Adolescence, 33, 247-260. doi:10.1023/B:JOYO.0000025323.94929.d9

Watson, P. J., Grisham, S. O., Trotter, M. V., \& Biderman, M. D. (1984). Narcissism and empathy: Validity evidence for the Narcissistic Personality Inventory. Journal of Personality Assessment, 45, 159-162. doi:10.1207/s15327752jpa4803_12

West, S., Taylor, A., \& Wu, W. (2012). Model fit and model selection in structural equation modeling. In R. Hoyle (Ed.), Handbook of structural equation modeling (pp. 209-231). New York, NY: The Guilford Press.

Wilson, M. S., \& Sibley, C. G. (2011). 'Narcissism creep?': Evidence for age-related differences in narcissism in the New Zealand general population. New Zealand Journal of Psychology, 40, 89-95.

Wink, P. (1991). Two faces of narcissism. Journal of Personality and Social Psychology, 61, 590-597. doi:10.1037/0022-3514.61.4.590 